\title{
TRES ESPECIES NUEVAS DE ENCARSIA (HYMENOPTERA: CHALCIDOIDEA: APHELINIDAE) DE MÉXICO
}

\author{
SVetlana N. MYARTSEVA, EnRiQue RUIZ-CANCINO \& JuAna María \\ CORONADO-BLANCO*
}

Facultad de Ingeniería y Ciencias, Universidad Autónoma de Tamaulipas, 87149 Cd. Victoria, Tamaulipas, México. *autora de correspondencia: <jmcoronado@uat.edu.mx>

Myartseva, S. N., Ruiz-Cancino, E. \& Coronado-Blanco, J. M. 2014. Tres especies nuevas de Encarsia (Hymenoptera: Chalcidoidea: Aphelinidae) de México. Acta Zoológica Mexicana (n. s.), 30(2): 288-297.

RESUMEN. La familia Aphelinidae es una de las más importantes en el control biológico de insectos plaga a nivel mundial, incluye 34 géneros y aproximadamente 1,300 especies, con 12 géneros y 184 especies en México. El género Encarsia es el más diverso con 409 especies y 94 en México. En el presente trabajo se describen e ilustran tres especies nuevas: Encarsia antennata sp. n. de Baja California Sur, E. caudata sp. n. de Nuevo León y E. oaxaca sp. n. de Oaxaca y Tamaulipas.

Palabras clave: Biodiversidad, parasitoides.

Myartseva, S. N., Ruiz-Cancino, E. \& Coronado-Blanco, J. M. 2014. Three new species of Encarsia (Hymenoptera: Chalcidoidea: Aphelinidae) from Mexico. Acta Zoológica Mexicana (n. s.), 30(2): 288-297.

ABSTRACT. Family Aphelinidae is one of the most important in the biological control of insect pests at world level. It includes 34 genera and around 1,300 species, with 12 genera and 184 species in Mexico. Genus Encarsia is the most diverse with 409 species and 94 in Mexico. In this paper, three new species of this genus are described and illustrated: E. antennata sp. n. from Baja California Sur, E. caudata sp. n. from Nuevo Leon, and E. oaxaca sp. n. from Oaxaca and Tamaulipas.

Key words: Biodiversity, parasitoids.

\section{INTRODUCCIÓN}

Los afelínidos son una pequeña familia de himenópteros chalcidoideos que contiene cerca de 1,350 especies en 36 géneros. Son cosmopolitas (Noyes 2013, Polaszek et al. 2013). Son pequeñas avispas con cuerpo de cerca de $1 \mathrm{~mm}$ de longitud y son reconocidos por la siguiente combinación de caracteres: antena de las hembras de tres a nueve segmentos, el número de los segmentos de la maza varía de cero a cuatro; mandíbula con dos a tres dientes y truncación; fórmula tarsal 5-5-5, 5-4-5 o 4-4-4: ala anterior con vena marginal larga; vena estigmal corta, vena postmarginal ausente o

Recibido: 23/10/2012; aceptado: 20/02/2014. 
corta; gaster con siete terguitos; tercer válvula separada y articulada con el segundo valvifer; macho similar a la hembra, grandemente confinado a la estructura de las antenas y genitalia. Son endoparasitoides o ectoparasitoides primarios o secundarios que atacan otros insectos, sus huevos u otros parasitoides (Polaszek 1991, Woolley 1997). Parasitan a una amplia variedad de especies dañinas a cultivos agrícolas y hortícolas (Hayat 1997). La mayoría de los hospederos de Aphelinidae pertenecen a cuatro familias de Hemiptera: Aleyrodidae, Diaspididae, Coccidae y Aphididae. Aphelinidae ha contribuido con más especies de enemigos naturales al éxito del control biológico que cualquier otra familia de insectos (Greathead 1986), las especies de Encarsia han jugado un papel sobresaliente en el combate de plagas de muchos países, incluyendo México (Myartseva \& Evans 2008).

Encarsia Förster es el género más diverso de la familia, contiene especies de importancia económica que son agentes de control biológico de insectos plaga, generalmente de mosquitas blancas (Aleyrodidae) y escamas armadas (Diaspididae) (Noyes 2013, Hayat 2012). El género está siendo estudiado en diversos países, por ejemplo, en China se registran 76 especies (Huang \& Polaszek 1998), en Australia 94 (Schmidt \& Polaszek 2007), en India 83 (Hayat 1998, 2012), en Estados Unidos 53 mientras que en Canadá sólo cinco especies han sido registradas (Noyes 2013). En México se introdujeron cuatro especies de Encarsia para combatir la mosca prieta de los cítricos Aleurocanthus woglumi Ashby (Myartseva \& Luna-Salas 2005). Recientemente, la importancia económica de Aphelinidae ha sido reconocida a nivel mundial, por lo que se ha puesto más atención al estudio de su biología y taxonomía. Dada la especificidad de hospederos y su enorme diversidad, las especies de Encarsia representan un recurso inagotable para el control biológico de escamas armadas y mosquitas blancas, pero la utilización efectiva de este recurso requiere de la identificación correcta de las especies, para lo cual se necesitan taxónomos con experiencia (Polaszek et al. 2009).

Para la identificación de las especies de Encarsia se usan las características morfológicas más importantes tales como la fórmula tarsal, la setación del disco alar y venación, dimensiones de los segmentos antenales, número de setas sobre el lóbulo medio del mesoescudo, distancia entre las sensilas placoidales escutelares y entre las setas largas escutelares, la relación de la longitud de la tibia media, las dimensiones de la longitud de la tercer válvula y segundo valvífer, la setación de los terguitos gastrales y la coloración del cuerpo.

En México se reportaron 88 especies de Encarsia en el libro "Género Encarsia de México. Una revisión, clave y descripción de nuevas especies" de Myartseva \& Evans (2008). Después fueron descritas otras especies en diferentes estados de México: E. escama Myartseva (Myartseva et al. 2008), E. madera Myartseva, E. mexicella Myartseva (Myartseva 2009) en Tamaulipas, E. morela Myartseva (Myartseva et al. 2010) en Morelos, E. clavata Myartseva \& González y E. verticina Myartseva \& 
González (Myartseva \& González-Hernández 2008) en Chiapas y Jalisco, E. tampicana Myartseva (Myartseva et al. 2011) en Veracruz, E. xilitla Myartseva en San Luis Potosí y E. yumka Myartseva (Myartseva et al. 2012b) en Tabasco. A pesar de la diversidad específica del género en México, se estima que el número total de las mismas podría ser de 1.5 a 2 veces el actual, debido a la alta diversidad de sus hospederos en el país, por lo que el presente trabajo tiene como objetivo la descripción de tres especies nuevas del género Encarsia encontradas en cuatro estados de México.

\section{MATERIAL Y MÉTODOS}

Los especímenes de Encarsia fueron prestados del Entomological Research Museum, University of California - Riverside Collection (UCRC), Estados Unidos (EUA). Se recibieron montados en triángulos de opalina y fueron montados en portaobjetos en bálsamo de Canadá, según el método de Noyes (1982) para la mejor observación de las características. Las medidas fueron tomadas de las divisiones de una escala lineal de un micrómetro colocado en el ocular de un microscopio compuesto Leica CME con magnificación de 400x. Dichos especímenes utilizados en el estudio fueron colectados durante la década de 1980 y 1990 en los estados de Baja California Sur, Nuevo León, Oaxaca y Tamaulipas (México). Se encuentran depositados en la colección del UCRC, Estados Unidos (EUA) y en el Museo de Insectos (MIFA) de la Universidad Autónoma de Tamaulipas, en Cd. Victoria, Tamaulipas, México.

\section{RESULTADOS}

\section{Encarsia antennata Myartseva, sp. $\mathrm{n}$.}

(Figs. 1-4)

Material. Holotipo hembra: México, Baja California Sur, Las Barracas, pan trap, 21.IV.1986 (col. P. DeBach). UCRC Ent. No 54435. Depositado en el Entomological Research Museum, University of California - Riverside, Estados Unidos.

Hembra. Longitud del cuerpo (sin el ovipositor): $0.8 \mathrm{~mm}$.

Coloración. Cabeza amarillo anaranjado. Antenas amarillas. Mesosoma anaranjado, lóbulos laterales amarillos, excepto el ápice. Alas anteriores hialinas, patas amarillas. Gáster anaranjado, último terguito amarillo, válvula 3 amarilla.

Estructura. Cabeza 1.3x tan ancha como alta. Frontovértice aproximadamente $0.6 \mathrm{x}$ tan ancho como la anchura de la cabeza. Distancia entre los ocelos posteriores ligeramente más corta que la distancia entre el ocelo posterior y el ojo. Ojos cerca de 1.4x tan largos como las mejillas. Antenas (Fig. 1) insertadas debajo del margen inferior de los ojos. Distancia entre tórulos cerca de 0.6x tan larga como la distancia del tórulo al ojo. Radícula antenal $2 \mathrm{x}$ tan larga como ancha. Escapo cerca de $4.4 \mathrm{x}$ tan largo como ancho. Pedicelo aproximadamente 1.4x tan largo como ancho. Segmento 


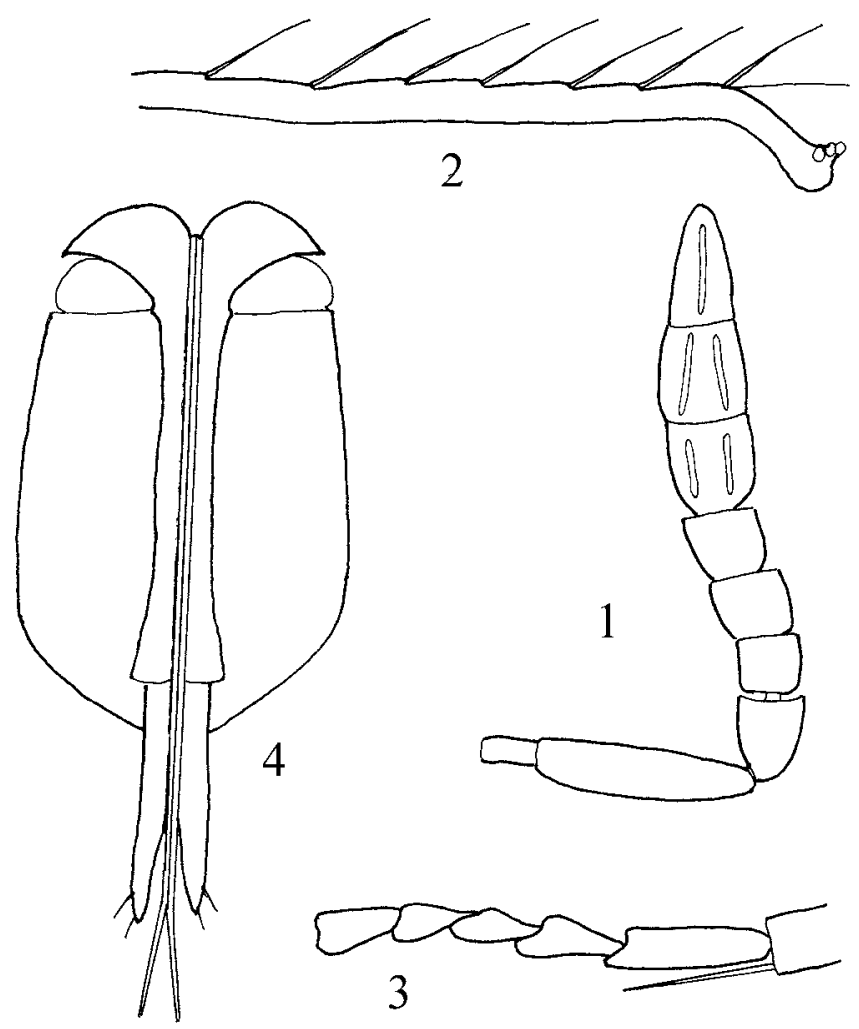

Figuras 1-4. Encarsia antennata Myartseva, sp. n., hembra: 1 - antena, 2 - detalle de la vena marginal, 3 - espuela de la tibia media y tarso medio, 4 - ovipositor en vista ventral.

funicular I subcuadrado, segmento II ligeramente más largo que ancho, segmento III subcuadrado. Funículo casi tan largo como el escapo (36:38). Maza antenal de tres segmentos, no más ancha que el funículo y más larga que el funículo y el pedicelo juntos. Lóbulo medio del mesoescudo con 14 setas delgadas, par basal de setas más robusto y cerca de $2 \mathrm{x}$ tan largo como las otras setas. Lóbulos laterales con tres setas largas cada uno, axilas con una seta cada una. Escutelo cerca de 0.6x tan largo como el lóbulo medio. Sensilas escutelares placoideas ampliamente espaciadas. Par anterior de setas escutelares visiblemente más cortas que el par posterior (17:23). Distancia entre las bases de las setas anteriores más o menos como la distancia entre las bases de las setas posteriores. Ala anterior uniformemente setosa, $3 \mathrm{x}$ tan larga como ancha, su fleco marginal ligeramente más de $0.3 x$ tan larga como la anchura alar máxima, base del ala con 14 setas cortas. Vena submarginal con tres setas largas, vena marginal (Fig. 2) con siete setas largas a lo largo del margen anterior. Fórmula tarsal 5-5-5. 
Espuela de la tibia media (Fig. 3) ligeramente más corta que el basitarso. Terguitos gástricos I-V con un grupo de pelos delgados en los lados de cada terguito, terguito VI con cuatro setas largas, terguito VII ligeramente elongado. Ovipositor expuesto (Fig. 4), 1.6x tan largo como la tibia media (sin los estiletes); válvula III cerca de $0.4 \mathrm{x}$ tan larga como el valvífer II.

Distribución. México (Baja California Sur).

Etimología. El nombre se refiere a la antena (que es más corta que lo normal).

Macho. Desconocido.

Comentarios taxonómicos. Encarsia antennata sp. n. es cercana a E. perplexa Huang \& Polaszek, se diferencia por las siguientes características: en E. antennata, el funículo es más o menos tan largo como el escapo, ala anterior hialina, $3 \mathrm{x}$ tan larga como ancha, su base con 14 setas, ovipositor 1.6x tan largo como la tibia media y la válvula III amarilla. En E. perplexa, el funículo es 1.3x tan largo como el escapo, ala anterior ahumada debajo de la vena marginal y 2.6x tan larga como ancha, su base con dos a tres setas, ovipositor 1.8-1.9x tan largo como la tibia media y los ápices de las válvulas III son negros. Se ha comparado con muchos especímenes de E. perplexa obtenidos en México de su hospedero Aleurocanthus woglumi Ashby (Aleyrodidae).

\section{Encarsia caudata Myartseva, sp. $\mathbf{n}$.}

(Figs. 5-8)

Material. Holotipo hembra: México, Nuevo León, Allende, Lazarillos de Abajo, 9.VII.1983 (col. A. González H.). UCRC Ent. No 54531. Depositado en el Entomological Research Museum, University of California - Riverside, Estados Unidos.

Hembra. Longitud del cuerpo: $0.5 \mathrm{~mm}$ (incluyendo el ovipositor).

Coloración. Cabeza y antenas amarillo claro, ocelos rojizos. Mesosoma amarillo claro, pronoto y margen anterior del lóbulo medio del mesoescudo ahumados. Alas anteriores hialinas, patas amarillo claro. Gáster amarillo claro, terguito V ahumado, terguitos VI y VII de pardo a negro.

Estructura. Cabeza no más ancha que el mesosoma, cerca de 1.2x tan ancha como alta. Frontovértice 0.6x tan ancho como la anchura de la cabeza. Cabeza, detrás de los ocelos posteriores, con estrías transversales delgadas. Distancia entre los ocelos posteriores cerca de $0.7 \mathrm{x}$ tan larga como la distancia del ocelo posterior al ojo. Ojos $1.5 \mathrm{x}$ tan largos como las mejillas. Mandíbula con tres dientes grandes y agudos. Antenas (Fig. 5) insertadas inmediatamente debajo del nivel del margen inferior de los ojos. Distancia entre tórulos $0.7 \mathrm{x}$ tan larga como la distancia del tórulo al ojo. Radícula antenal cerca de 2x tan larga como ancha. Escapo 5x tan largo como ancho. Pedicelo $1.3 \mathrm{x}$ tan largo como ancho. Segmento funicular I cerca de $2 \mathrm{x}$ tan largo como ancho $\mathrm{y}$ más o menos tan largo como el pedicelo (11:12); segmento II es 1.4x tan largo como ancho; segmento III es $1.7 \mathrm{x}$ tan largo como ancho. Maza antenal de tres segmentos, ligeramente más ancha que el funículo y ligeramente más corta que el funículo y el 


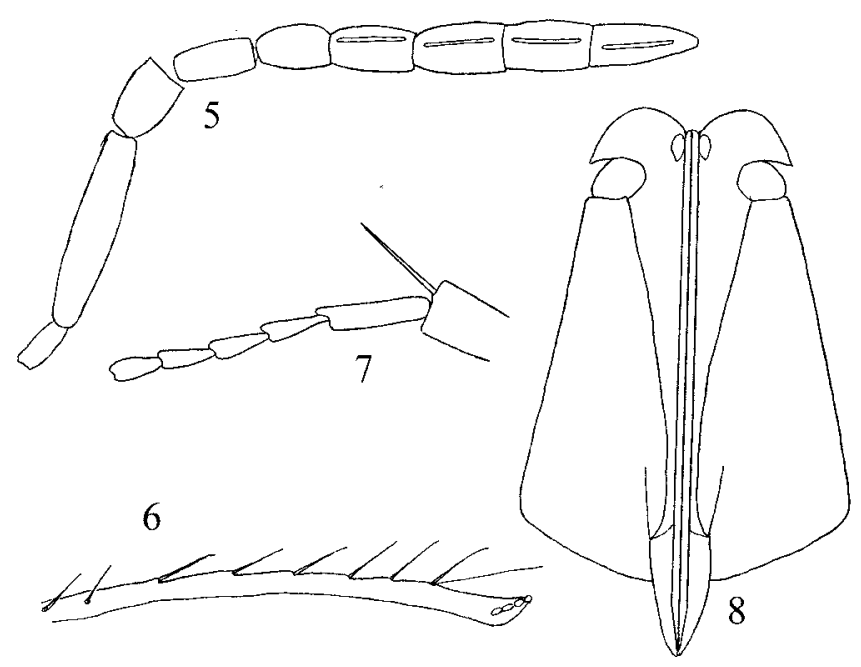

Figuras 5-8. Encarsia caudata Myartseva, sp. n., hembra: 5 - antena, 6 - detalle de la vena marginal, 7 - espuela de la tibia media y tarso medio, 8 - ovipositor en vista ventral.

pedicelo juntos. Segmento funicular III y segmentos de la maza con una sensila longitudinal cada uno. Lóbulo medio del mesoescudo con cuatro pares de setas situadas simétricamente; par basal de setas más largo y fuerte. Lóbulos laterales con dos setas cada uno, axilas con una seta cada una. Escutelo cerca de 1.8x tan ancho como largo. Sensilas escutelares placoideas pequeñas y redondas, separadas por una distancia de 5 diámetros de una sensila. Distancia entre las bases de las setas escutelares anteriores cerca de $0.5 \mathrm{x}$ tan larga como la distancia entre las bases de las setas posteriores. Par anterior de setas $0.4 \mathrm{x}$ tan largo como el par posterior. Alas anteriores uniformemente setosas, cerca de 3x tan largas como la anchura alar máxima, su fleco marginal aproximadamente $0.5 \mathrm{x}$ tan largo como la anchura del ala, base con cuatro setas. Vena marginal (Fig.6) con seis setas a lo largo del margen anterior. Fleco marginal del ala posterior más largo que la anchura alar máxima. Fórmula tarsal 5-5-5. Espuela de la tibia media (Fig. 7) más o menos tan larga como el basitarso. Ovipositor (Fig. 8) no expuesto, su longitud cerca de $1.2 \mathrm{x}$ tan largo como la tibia media; válvula III es $0.25 \mathrm{x}$ tan larga como el valvífer II.

Distribución. México (Nuevo León).

Etimología. El nombre se refiere al gáster (el ápice es negro y el resto del cuerpo es amarillo).

Macho. Desconocido.

Comentarios taxonómicos. Encarsia caudata sp. n. es cercana a E. pitilla Myartseva $\&$ Evans, descrita del Estado de Tamaulipas, se distinguen en las características 
siguientes: en E. caudata, los terguitos apicales son de pardo a negro, maza antenal más larga que el funículo y el pedicelo juntos, segmento flagelar II sin sensila, lóbulo medio del mesoescudo con ocho setas, base del ala anterior con cuatro setas y la vena marginal con seis setas a lo largo del margen anterior. En E. pitilla, gáster con manchas ahumadas en los lados exteriores de los terguitos I-IV, maza antenal ligeramente más larga que el funículo, segmento flagelar II con sensila lineal, lóbulo medio del mesoescudo con cuatro a seis setas, base del ala anterior con 9-12 setas y la vena marginal con 8-10 setas a lo largo del margen anterior. El holotipo de E. pitilla se ha estudiado, está depositado en el MIFA-UAT.

\section{Encarsia oaxaca Myartseva, sp. n.}

(Figs. 9-12)

Material. Holotipo hembra: México, Oaxaca, Yagul Ruins, 13.VII.1984 (col. G. Gordh). UCRC Ent. No 54558. Paratipos (2): México, Tamaulipas, $3 \mathrm{~km}$ S Ciudad Victoria, "Hwy 5, screen sweeping", 1 ㅇ, 1.XI.1982 (coll. J.T. Huber \& A. González), UCRC Ent. No 54325; Ciudad Victoria, ex Diaspididae en Nerium oleander, 1 9 , 25.VIII.1998 (col. S.N. Myartseva). Holotipo y un paratipo depositados en la colección UCRC; un paratipo en el Museo de Insectos (MIFA).

Hembra. Longitud del cuerpo: 0.6-0.7 mm (incluye ovipositor).

Coloración. Cabeza parda, antenas amarillas. Mesosoma pardo, lóbulos laterales amarillos excepto los ápices. Alas anteriores hialinas. Patas amarillas. Gáster con los

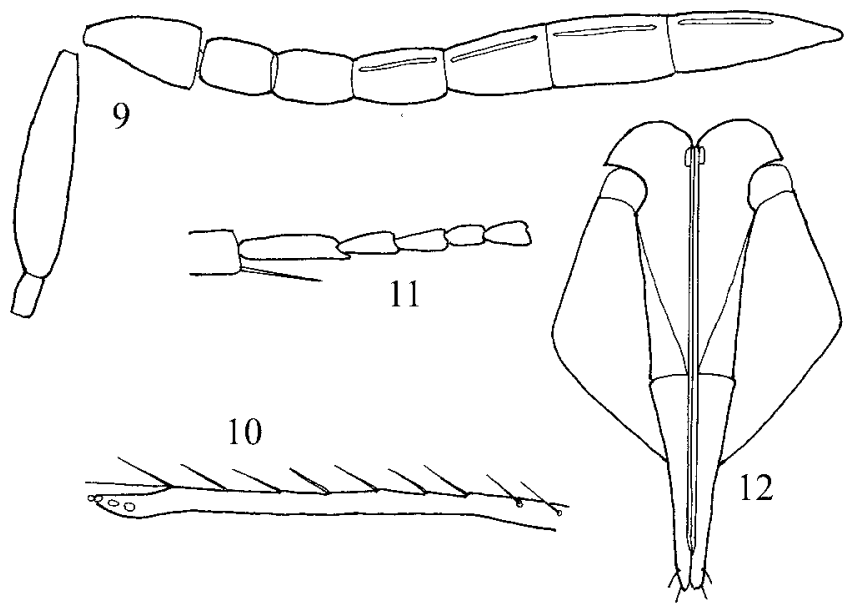

Figuras 9-12. Encarsia oaxaca Myartseva, sp. n., hembra: 9 - antena, 10 - detalle de la vena marginal, 11 - espuela de la tibia media y tarso medio, 12 - ovipositor en vista ventral. 
terguitos I-IV y VII amarillos, terguitos V y VI parduzcos. Válvulas III amarillas, ápices de los estiletes negros.

Estructura. Cabeza 1.2-1.3x tan ancha como su altura. Frontovértice 0.6x tan ancho como la anchura de la cabeza. Distancia entre los ocelos posteriores cerca de $1.5 \mathrm{x}$ más corta que la distancia del ocelo posterior al ojo. Ojos setosos, cerca de 1.3x tan largo como las mejillas. Antenas (Fig. 9) insertadas inmediatamente debajo del nivel del margen inferior de los ojos. Distancia entre tórulos cerca de $1.5 \mathrm{x}$ más corta que la distancia del tórulo al ojo. Radícula antenal cerca de $2 \mathrm{x}$ tan larga como ancha. Escapo 4.0-4.2x tan largo como ancho. Pedicelo cerca de 1.8x tan largo como ancho. Segmento funicular I cerca de 1.3x tan largo como ancho; segmento II ligeramente más largo y $1.5 \mathrm{x}$ tan largo como ancho; segmento III ligeramente más largo y más ancho que el segmento II y cerca de 1.6x tan largo como ancho. Maza antenal de tres segmentos, ligeramente más ancha que el funículo y más larga que el funículo y el pedicelo juntos (60:65); segmento apical de la maza adelgazado hacia el ápice y visiblemente más largo que el segmento precedente. Lóbulo medio del mesoescudo y axilas con escultura reticulada ancha, lóbulo medio con ocho setas largas situadas simétricamente (variabilidad: un especimen con nueve setas). Lóbulos laterales con tres setas cortas cada uno, axilas con una seta corta cada una. Escutelo con escultura reticulada transversal en el tercio anterior, cerca de $0.7 \mathrm{x}$ tan largo como el lóbulo medio y cerca de $2 \mathrm{x}$ tan ancho como largo. Sensilas escutelares placoideas ovales y ampliamente espaciadas. Distancia entre las bases de las setas escutelares anteriores ligeramente más larga que la distancia entre las bases de las setas posteriores. Setas posteriores $1.3 \mathrm{x}$ tan largas como las anteriores. Alas anteriores uniformemente setosas, 2.5-3x tan largas como la anchura alar máxima, su fleco marginal cerca de $0.3 \mathrm{x}$ tan largo como la anchura del ala, base con cuatro setas cortas. Vena marginal (Fig. 10) con seis a siete setas a lo largo del margen anterior. Fórmula tarsal 5-5-5. Espuela de la tibia media (Fig. 11) más o menos tan larga como el basitarso. Terguitos gástricos II-VII con 2, 2, 2, 4, 2 y 4 setas, respectivamente. Ovipositor (Fig. 12) ligeramente expuesto, tan largo como la tibia media; válvula III ligeramente más corta que el valvífer II (30:35).

Distribución. México (Oaxaca y Tamaulipas).

Etimología. El nombre se refiere al estado de Oaxaca en que fueron recolectados los ejemplares.

Macho. Desconocido.

Comentarios taxonómicos. Encarsia oaxaca n. sp. es cercana a E. juanae Myartseva $\&$ Evans, descrita del Estado de Tamaulipas y obtenida de Diaspididae; se distinguen por las características siguientes: en E. oaxaca los segmentos funiculares son más largos, 1.3, 1.5 y 1.6x tan largos como anchos, respectivamente, y la maza antenal visiblemente más larga que el funículo y el pedicelo juntos, ovipositor tan largo como la tibia media, válvula III ligeramente más corta que el valvífer II, mesosoma pardo y 
la mitad basal del gáster es amarilla. En E. juanae los segmentos funiculares son más cortos, 1.1, 1,1 y 1.2x tan largos como anchos, respectivamente, y la maza antenal subigual o más corta que el funículo y el pedicelo juntos, ovipositor ligeramente más corto que la tibia media, válvula III es $0.48 x$ tan larga como el valvífer II, mesosoma amarillo con un área triangular central parduzca y la mitad basal del gáster es parda. El holotipo de E. juanae se ha estudiado, está depositado en el MIFA-UAT.

Las tres especies de Encarsia pueden ser ubicadas en las claves de Myartseva et al. (2012a) en los siguientes pares, con E. perplexa (par 66) se compara a Encarsia antennata, con E. pitilla (par 94) se compara a E. caudata y con E. juanae (par 93) se compara a E. oaxaca.

AGRADECIMIENTOS. Al proyecto PROMEP "Taxonomía y ecología de fauna y micobiota en comunidades forestales y cultivos de México", de la Red de Cuerpos Académicos y a la UAT, por su apoyo para la realización de este trabajo.

\section{LITERATURA CITADA}

Greathead, D. 1986. Parasitoids in classical biological control, pp. 289-318. In: J. Waage and D. Greathead (Eds.). Insect parasitoids. Academic Press, London.

Hayat, M. 1998. Aphelinidae of India (Hymenoptera: Chalcidoidea): a taxonomic revision. Memoirs on Entomology, International. Associated Publishers, Gainesville, Florida, U.S.A. 416 pp.

Hayat, M. 2012. Additions to the Indian Aphelinidae (Hymenoptera: Chalcidoidea) - III: the genus Encarsia Förster. Oriental Insects, 45: 202-274.

Huang, J. \& Polaszek, A. 1998. A revision of the Chinese species of Encarsia Foerster (Hymenoptera: Aphelinidae): parasitoids of whiteflies, scale insects and aphids (Hemiptera: Aphelinidae, Diaspididae, Aphidoidea). Journal of Natural History, 32: 1825-1966.

Myartseva, S. N. 2009. Two new species of Encarsia (Hymenoptera: Aphelinidae), parasitoids of whiteflies (Homoptera: Aleyrodidae) from Mexico. Biosystematica, 3: 27-31.

Myartseva, S. N. \& Evans, G. A. 2008. Genus Encarsia Förster of Mexico (Hymenoptera: Chalcidoidea: Aphelinidae). A revision, key and description of new species. Serie: Avispas Parasiticas de Plagas y Otros Insectos No. 3. Universidad Autónoma de Tamaulipas, Ciudad Victoria, México. $320 \mathrm{pp}$.

Myartseva, S. N. \& González-Hernández, A. 2008. Descripciones de dos especies nuevas de Encarsia (Hymenoptera: Aphelinidae) y nuevos registros de especies de este género para Chiapas y Jalisco, México. Acta Zoológica Mexicana (n.s.), 24: 101-114.

Myartseva, S. N. \& Luna-Salas, J. F. 2005. Encarsia perplexa Huang \& Polaszek, 1998 (Hymenoptera: Chalcidoidea, Aphelinidae) en México y el Sureste de Texas, EUA. Folia Entomológica Mexicana, 44: 297-304.

Myartseva, S. N., Ruiz-Cancino, E. \& Coronado-Blanco, J. M. 2008. Encarsia aurantii speciesgroup (Hymenoptera: Aphelinidae), parasitoids of armored scales (Hemiptera: Diaspididae) in Mexico, with key and description of a new species. Zoosystematica Rossica, 7: 67-71.

Myartseva, S. N., Ruiz-Cancino, E., Coronado-Blanco, J. M. \& Corona-López, A. M. 2010. Especies de Encarsia (Hymenoptera: Aphelinidae) que parasitan Trialeurodes vaporariorum (Westwood) (Hemiptera: Aleyrodidae) en Tamaulipas y Morelos, México, y descripción de una especie nueva. Dugesiana, 17: 129-135. 
Myartseva, S. N., Ruiz-Cancino, E. \& Coronado-Blanco, J. M. 2011. Nueva especie de Encarsia Foerster y nuevos registros de avispas parasíticas (Chalcidoidea: Aphelinidae, Encyrtidae) de mosquitas blancas (Hemiptera: Aleyrodidae) de Veracruz, México. Acta Zoológica Mexicana (n.s.), 27: 803-809.

Myartseva S. N., Ruiz C., E.. \& Coronado B., J. M. 2012a. Aphelinidae (Hymenoptera) de importancia agrícola en México. Revisión y claves. UAT. México. 412 pp.

Myartseva, S. N., Ruiz-Cancino, E. \& Coronado-Blanco, J. M. 2012b. Dos nuevas especies de Encarsia Foerster (Hymenoptera: Aphelinidae) de los estados de San Luis Potosí y Tabasco, México. Acta Zoológica Mexicana (n.s.), 28: 457-464.

Noyes, J. S. 1982. Collecting and preserving chalcid wasps (Hymenoptera: Chalcidoidea). Journal of Natural History, 16: 315-334.

Noyes, J. S. 2013. Universal Chalcidoidea Database. www.nhm.ac.uk/entomology/ chalcidoids/index. html (última actualización: Octubre 2013).

Polaszek, A. 1991. Egg parasitism in Aphelinidae (Hymenoptera: Chalcidoidea) with special reference to Centrodora and Encarsia species. Bulletin of Entomological Research, 81: 97-106.

Polaszek, A., Hernández-Suárez, E. M., Mansari, S., Pedata, P. A. \& Schmidt, S. 2009. Megadiversity of Encarsia (Chalcidoidea, Aphelinidae): macroevolution in a microhymenopteran, pp. 81-86. In: E. Ruiz C. \& J.M. Coronado B. (Eds.). Taller Internacional de Recursos Naturales. Memoria. México.

Polaszek, A., Ayshford, T., Yahya, B. E. \& Fusu, L. 2013. Wallaceaphytis: an unusual new genus of parasitoid wasp (Hymenoptera: Aphelinidae) from Borneo. Journal of Natural History, DOI: 10.1080/00222933.2013.852264.

Schmidt, S. \& Polaszek, A. 2007. The Australian species of Encarsia Förster (Hymenoptera, Chalcidoidea: Aphelinidae), parasitoides of whiteflies (Hemiptera, Sternorrhyncha, Aleyrodidae) and armoured scale insects (Hemiptera, Coccoidea: Diaspididae). Journal of Natural History, 41: 20992265.

Woolley, J. B. 1997. Aphelinidae, pp. 134-150. In: G. A. P., Gibson, J. T., Huber \& J. B., Woolley (Eds.). Annotated keys to the genera of Nearctic Chalcidoidea (Hymenoptera). NRC Research Press, Ottawa, Canada. 794 pp. 